Laurence Guyard, Aurélia Mardon, éds, Le corps à l'épreuve du genre, entre normes et pratiques

Nancy, Presses universitaires de Nancy, coll. Épistémologie du corps, 2010

\title{
Aurélie Olivesi
}

\section{(2) OpenEdition}

\section{Journals}

Édition électronique

URL : http://journals.openedition.org/questionsdecommunication/2854

DOI : 10.4000/questionsdecommunication.2854

ISSN : 2259-8901

Éditeur

Presses universitaires de Lorraine

Édition imprimée

Date de publication : 30 juin 2011

Pagination : 380-382

ISBN : 978-2-8143-0084-2

ISSN : 1633-5961

Référence électronique

Aurélie Olivesi, « Laurence Guyard, Aurélia Mardon, éds, Le corps à l'épreuve du genre, entre normes et pratiques ", Questions de communication [En ligne], 19 | 2011, mis en ligne le, consulté le 22 septembre 2020. URL : http://journals.openedition.org/questionsdecommunication/2854 ; DOI : https://doi.org/ 10.4000/questionsdecommunication.2854

Ce document a été généré automatiquement le 22 septembre 2020

Tous droits réservés 


\section{Laurence Guyard, Aurélia Mardon, éds, Le corps à l'épreuve du genre, entre normes et pratiques}

Nancy, Presses universitaires de Nancy, coll. Épistémologie du corps, 2010

\section{Aurélie Olivesi}

\section{RÉFÉRENCE}

Laurence Guyard, Aurélia Mardon, éds, Le corps à l'épreuve du genre, entre normes et pratiques, Nancy, Presses universitaires de Nancy, coll. Épistémologie du corps, 2010, $191 \mathrm{p}$.

1 L'ouvrage collectif Le corps à l'épreuve du genre, entre normes et pratiques, édité par Laurence Guyard et Aurélia Mardon, s'attache à montrer à quel point les différences physiques entre hommes et femmes se voient réinscrites dans l'ordre social du genre au sens de gender, le sexe en tant qu'il est socialement construit. Les 11 contributions croisent les approches historique, sociologique et ethnographique afin d'approfondir la compréhension des mécanismes de la construction genrée des corps et, par là même, de comprendre comment des individus intériorisent des représentations, des normes et des pratiques corporelles sexuellement différenciées et hiérarchisées - ou y résistent. L'introduction de l'ouvrage réorganise les trois grandes approches thématiques (l'éducation et la socialisation, la médecine et le monde du travail) selon trois problématiques: la construction naturalisée de la féminité et de la virilité, la socialisation et à la mise en scène genrée des corps et les modalités et temporalités d'apprentissage des habitudes corporelles sexuées - avec les injonctions paradoxales qu'elles mettent en œuvre.

2 La première partie de l'ouvrage, intitulée «Corps, genre et âges de la vie », s'ouvre sur un texte de Geneviève Cresson consacré à la prise en compte de la question du sexe/ 
genre dans la vie quotidienne des enfants dans les crèches à partir d'une observation participante dans les crèches de la région lilloise. Ainsi l'auteure observe-t-elle une différence entre le discours des professionnels - selon lequel les besoins des enfants sont considérés comme similaires et les pratiques indifférenciées - et son observation, qui montre des registres sexués (et hiérarchisés) de perception, de description et d'observation des enfants, à travers lesquels les enfants sont familiarisés avec le genre avant même d'apprendre à repérer les sexes. L'article de Martine Court sur les processus de socialisation, qui provoquent le rejet du travail de l'apparence à l'adolescence, s'appuie sur la technique du portrait individuel d'un cas surprenant du point de vue sociologique : une jeune fille de 11 ans dont le rapport à l'apparence est particulièrement peu conforme aux stéréotypes de genre - alors même que sa mère est esthéticienne et que sa sœur aînée accorde une grande attention à son apparence. À l'encontre des discours communs qui naturalisent ces conduites non conformes, cette analyse met en lumière trois processus de socialisation essentiels: la rareté des contacts avec d'autres jeunes filles de son âge, une distance aux modèles féminins proposés par les médias et l'exposition au sein de la famille à des discours critiques voire violents - sur le travail de l'apparence. De la même manière, à travers une double méthodologie constituée, à la fois, d'entretiens auprès de collégiennes, de leurs parents et de protagonistes du monde scolaire et d'une observation dans les classes et la cour de récréation, Aurélia Mardon montre comment l'adolescence constitue une période charnière dans l'intériorisation par les jeunes filles de l'injonction paradoxale les poussant à mettre en valeur leur corps tout en faisant preuve de modération. Cette " féminité respectable " - norme se construisant presque exclusivement dans le regard des garçons -, se constitue au croisement des codes vestimentaires de leurs pairs et du contrôle des adultes. Même si les filles peuvent contourner ces injonctions et, avec l'âge, s'y opposer franchement à travers leurs styles vestimentaires, une telle éducation à l'apparence contribue indirectement à reproduire la dichotomie entre «filles bien » et « filles faciles».

3 La prééminence du regard masculin est également soulignée par Annie Ferrand dans une analyse qualitative de quinze manuels d'éducation des préadolescents et adolescents à la sexualité et aux identités de sexe qui en souligne l'androcentrisme et en dégage des éléments invariants, comme les erreurs, les inexactitudes ou l'usage de mots connotés et chargés de valeur morale pour décrire la biologie. L'invisibilisation du sexe et de la sexualité de femmes révèle une hiérarchisation à plusieurs échelons entre actif/passif, sujet/objet, performant/inerte qui fonctionne comme un «mode d'emploi de la domination ». Colin Giraud montre dans quelle mesure le corps est à la fois un support, un révélateur et un indicateur empirique des normes spécifiques aux « lieux gays " à Paris et Montréal, mettant en œuvre une relation tripartite entre espaces, corps et identités sociales. Loin d'être des enclaves de liberté ou des espaces sociaux anormaux et non contraints dans lesquels s'épanouirait une sexualité protégée du regard dominant, ces lieux constituent, au contraire, des scènes sociales où l'apparence et le jugement sur cette apparence structurent l'espace et les comportements - même si l'incorporation de ces normes varie beaucoup selon les milieux sociaux et les contextes biographiques de ceux qui les fréquentent.

4 La deuxième partie de l'ouvrage s'attache aux rapports entre le corps et le genre dans la médecine. Muriel Salle adopte une perspective historique pour souligner combien la passion du XIXe siècle pour l'hermaphrodisme se nourrit de la conquête de la légitimité professionnelle et sociale des médecins qui deviennent seuls habilités à prononcer une 
éventuelle réassignation du patient - réassignation lourde de conséquences en un temps où l'appartenance à un sexe plutôt qu'à un autre a de telles implications en termes de droits individuels, sociaux et politiques (le plus grand risque étant qu'un homme soit pris pour une femme). C'est à la pratique sportive féminine entre 1880 et 1922 que s'intéresse Anaïs Bohuon à travers une analyse multifocale de plus de 200 textes médicaux et scientifiques qui ont pour fondement commun une volonté de contrôler les corps féminins et la santé de la population, au point que la pratique physique féminine peut être pensée comme une «noso-politique » dans une volonté de maximaliser les corps féminins tout en introduisant une norme de santé féminine différente de celle des hommes. De son côté, Laurence Guyard formule l'hypothèse que l'intrusion de normes esthétiques dans le cadre de la consultation gynécologique serait liée à la féminisation de cette spécialité médicale, qu'elle vérifie dans une double méthodologie : l'observation de consultations gynécologiques et des entretiens auprès de gynécologues femmes et hommes.

Consacrée au monde professionnel, la troisième partie de l'ouvrage s'ouvre sur une enquête qualitative (entretiens et observation in situ) menée par Jeanne Teboul dans plusieurs organismes militaires de la chaîne « recrutement » de l'armée de terre durant 2 ans. L'auteure montre dans quelle mesure la neutralité sexuée demandée aux recrues féminines paraît correspondre au modèle de la masculinité hégémonique, et leur socialisation militaire constitue un changement symbolique de sexe. Dans le cas de l'entretien d'embauche, Oumaya Hidri a analysé (par une observation non participante de 17 entretiens et les entretiens ethnographiques des 7 recruteurs de cadres commerciaux observés) comment les trajectoires de vie des recruteurs jouent comme des grilles implicites d'évaluation des candidats aux professions de cadre commercial. En interrogeant la question de l'apparence physique, l'auteure s'attache au rapport entre construction genrée des corps et la proximité sociale ressentie par les recruteurs répartis en trois profils-types (ceux qui se reconnaissent dans les candidats, ceux qui partagent le même bagage social et ceux qui souhaitent qu'ils se conforment à un rôle) qui font de l'entretien d'embauche le lieu d'expression privilégié d'un cumul de discriminations. A contrario, c'est à des femmes achevant leur carrière dans des emplois de bureaux (subalternes et féminisés) que s'est intéressée Marjolaine Roger pour montrer à quel point les femmes de plus de 50 ans occupant ces emplois subissent un phénomène paradoxal : alors que leurs corps subissent une « invisibilisation » sociale et professionnelle, ils sont en même temps rendus très disponibles.

6 Face à la richesse des contributions de cet ouvrage et aux perspectives très éclairantes qu'elles soulèvent, on en vient, parfois, à regretter le moindre poids consacré au point de vue masculin. Mais il s'agit du point d'achoppement traditionnel des études sur le genre dont la cause est à chercher du côté de la valence différentielle des genres plutôt que dans des carences de l'analyse. 


\section{AUTEURS}

AURÉLIE OLIVESI

LERASS, université Paul Valéry-Montpellier 3

aurelie.olivesi@gmail.com 